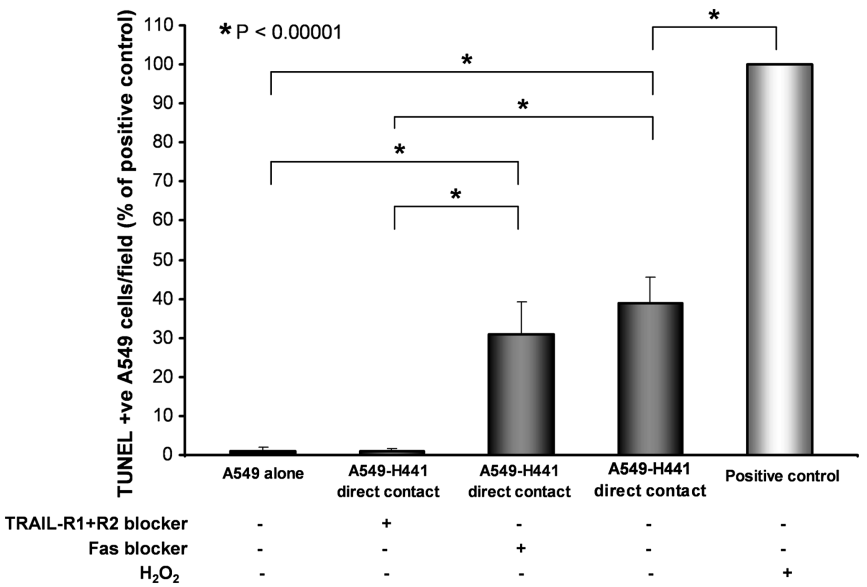

Abstract S138 Figure $1 \mathrm{H} 441$ direct contact induced apoptosis in A549 cells through TRAIL-R1 +R2 (10 $\mu \mathrm{g} / \mathrm{ml}$ each) blockade. Fas blocker $(10 \mu \mathrm{g} / \mathrm{ml})$ failed to block direct cell contact- induced apoptosis. In positive control, apoptosis was induced with $200 \mu \mathrm{M}$ H2O2. Negative control was represented by A549 cells cultured alone in monolayer.

Methods Using an in vitro wound repair model we explored the interaction of human Clara cells (H441 cell line) and type II AEC (A549 cell line). A transwell co-culture system was developed to determine the direct contact effect of densely populated Clara cells on wounded AEC monolayers.

Results In serum-free media, lone $\mathrm{H} 441$ cell wound repair was higher than equivalent A549 cells, despite the fourfold slower doubling time of H441 cells. Serum-free conditioned media obtained from unwounded and wounded H441 monolayers did not show any significant influence on A549 wound repair. However, in a direct contact coculture A549-H441 cell model significant inhibition of A549 wound repair $(p<0.005)$ was observed. Interestingly, H441 migration into the injured A549 layer was seen after $24 \mathrm{~h}$; with a significant proportion of migrated H441 cells found at the wound margins. Coupled to this migration we observed a $50 \%$ reduction in A549 cell number at the wound margins. TUNEL assay detected about 40\% A549 apoptosis in juxta-wound monolayers in A549-H441 direct contact ( $\mathrm{p}<0.00001$ ). This direct contact-induced apoptosis was significantly blocked by TRAIL-R1 and R2 combined receptor blockers ( $p<0.00001)$; whereas, Fas blocker failed to block this apoptosis.

Conclusion In summary, direct contact of H441 cells induces apoptosis in the A549 monolayers through a TRAIL-dependent mechanism which disrupts wound margin integrity, inhibiting wound repair. This novel observation warrants further exploration of the role of Clara cell-alveolar epithelial cell interaction within the context of aberrant wound repair associated with chronic fibrotic lung disorders.

\section{S139 THE $\mathrm{K}^{+}$CHANNEL KCa3.1 IS A NOVEL TARGET FOR THE TREATMENT OF IDIOPATHIC PULMONARY FIBROSIS}

doi:10.1136/thx.2010.150946.40

${ }^{1} \mathrm{M}$ C Shepherd, ${ }^{1} \mathrm{~S}$ R Sabir, ${ }^{1} \mathrm{C}$ McSharry, ${ }^{2} \mathrm{H}$ Wulff, ${ }^{3} \mathrm{P}$ Bradding. ${ }^{1}$ Division of Immunology, Infection and Inflammation, University of Glasgow, Glasgow, UK; ${ }^{2}$ Department of Pharmacology, University of California, Davis, USA; ${ }^{3}$ Institute for Lung Health, Department of Infection, Immunity and Inflammation, University of Leicester., Leicester, UK

Introduction and objectives Idiopathic pulmonary fibrosis (IPF) is common, largely unresponsive to treatment with a median survival of 3 years. New therapies are urgently required. IPF is characterised by proliferation of pulmonary mesenchymal cells through epithelial mesenchymal transition, resident fibroblast proliferation and circu- lating fibrocyte recruitment. We have previously demonstrated that the potassium channel $\mathrm{K}_{\mathrm{Ca}} 3.1$ regulates lung mesenchymal cell proliferation, is up-regulated by TGF $\beta$, an important driver of IPF, and is present in fibrocytes in peripheral blood. We tested the hypotheses that $\mathrm{K}_{\mathrm{Ca}} 3.1$ is up-regulated in IPF using the bleomycininduced pulmonary fibrosis murine model and that $\mathrm{K}_{\mathrm{Ca}} 3.1$ inhibition reduces pulmonary fibrosis.

Methods Prophylactic (Day - 3 ) and daily thereafter, sub-cutaneous TRAM-34, a specific $\mathrm{K}_{\mathrm{Ca}} 3.1$ inhibitor, was administered to C57BL/6 mice later exposed to nasal bleomycin (Day 0) and culled on day +21. Mice exposed to PBS or bleomycin acted as negative and positive controls. The primary endpoint was histological fibrosis score. Inflammation was assessed by bronchoalveolar lavage. Collagen deposition and $\mathrm{K}_{\mathrm{Ca}} 3.1$ expression were assessed by Masson's trichrome staining and qPCR.

Results Bleomycin-induced pulmonary fibrosis characterised by thickened alveolar septae, architectural destruction and collagen deposition. Co-administration of TRAM-34 significantly reduced pulmonary fibrosis (Modified Ashcroft's score +/- SEM: 4.8+/-0.8 bleomycin group vs $2.6+/-0.6$ TRAM-34 group: $p=0.02)$. Bleomycin increased lung $\mathrm{K}_{\mathrm{Ca}} 3.1$ (55-fold versus $\mathrm{PBS}$ control) and collagen I $\alpha$ mRNA (fourfold) expression ( $\mathrm{n}=3$ in each case). Mice receiving bleomycin lost more weight ( $2.39 \mathrm{vs} 0 \mathrm{~g}$ ) and had greater mortality than those co-administered TRAM-34. BAL cellularity did not differ between the groups. Collagen staining was reduced in the TRAM-34 group.

Conclusions $\mathrm{K}_{\mathrm{Ca}} 3.1$ expression is increased in a model of pulmonary fibrosis and inhibition with TRAM-34 significantly improves pathological outcome. The mechanism is likely to involve the modulation of cells involved in the fibrotic process. Previous clinical studies have shown KCa3.1 inhibition to be safe in humans and our study provides a rationale for a clinical trial of $\mathrm{K}_{\mathrm{Ca}} 3.1$ inhibitors in human IPF.

\section{TRAul34reduses ing fitrosis soore in tlearmaintested mice}

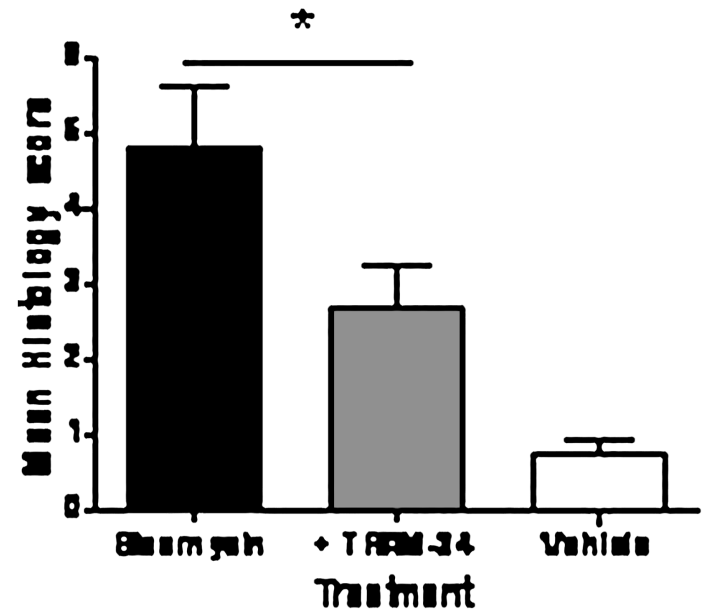

Abstract S139 Figure 1

\section{S140 THE ROLE OF TRANSFORMING GROWTH FACTOR- $\beta$ ACTIVATED KINASE-1 (TAK-1) IN THE DEVELOPMENT OF AIRWAY FIBROSIS}

doi:10.1136/thx.2010.150946.41

A Gardner, M J Brodlie, D A Mann, L A Borthwick, A J Fisher. Institute of Cellular Medicine, Newcastle University, Newcastle upon Tyne, UK

Introduction and aims Fibrotic disorders of the lung are characterised by an increase in fibroblast numbers and excessive deposition of extra 
cellular matrix, resulting in loss of airway or alveolar function. Murine lineage tracing experiments have suggested that these fibroblasts may, in part, be derived from lung epithelium via epithelial to mesenchymal transition (EMT). Transforming Growth Factor- $\beta 1$ (TGF- $\beta 1$ ) has been shown to drive EMT both in vitro and in vivo. Previous work in our lab has shown that inflammatory stimuli such as Tumour Necrosis Factor- $\alpha(T N F \alpha)$ can accentuate TGF- $\beta 1$ driven EMT in primary bronchial epithelial cells (PBEC). This crosstalk between inflammatory and TGF- $\beta 1$ signalling may have implications in the pathogenesis of fibrotic disorders of the lung including Bronchiolitis Obliterans Syndrome and Idiopathic Pulmonary Fibrosis. We hypothesised that TGF- $\beta$ activated kinase-1 (TAK-1), a protein phosphorylated in response to both TGF- $\beta 1$ and TNF $\alpha$, may be a key convergent point and assessed its role in inflammatory accentuation of EMT.

Methods PBEC isolated from stable lung transplant recipients were treated with TGF- $\beta 1(10 \mathrm{ng} / \mathrm{ml})$ and/or TNF $\alpha(20 \mathrm{ng} / \mathrm{ml})$ and the phosphorylation and localisation of signalling molecules SMAD-3 and TAK-1 assessed (western blot, confocal microscopy). TAK-1 function was blocked using a selective inhibitor and by siRNA knockdown; effects on EMT marker expression were assessed (western blot, confocal microscopy).

Results TAK- 1 is phosphorylated in response to both TGF- $\beta 1$ (161\% increase) and TNFa (145\%) with an accentuated phosphorylation upon co-treatment $(516 \%, n=3)$. Co-treatment also resulted in pronounced nuclear localisation of phosphorylated TAK-1, which has not previously been reported. Chemical Inhibition of TAK-1 phosphorylation significantly reduced TGF- $\beta 1$ driven down-regulation of E-cadherin ( $22 \%$ inhibition) and up-regulation of Vimentin $(78 \%)$ and Fibronectin (39\%, $\mathrm{p}<0.05, \mathrm{n}=4)$. Furthermore, TAK-1 inhibition significantly reduced TNF $\alpha$ accentuated TGF- $\beta 1$ driven down-regulation of E-cadherin (39\%) and up-regulation of Fibronectin $(87 \%)$ and Vimentin $(258 \%, \mathrm{p}<0.05, \mathrm{n}=4)$. These findings were independently validated via siRNA knockdown of TAK-1.

Conclusions These findings suggest that TAK- 1 is an important convergent point for inflammatory and TGF- $\beta 1$ signalling, controlling the accentuation of EMT in primary human airway epithelium. Examination of TAK-1 activation and function in animal models of lung fibrosis may provide information on the potential for TAK-1 inhibition as a therapeutic target.

\section{S141 A DISINTEGRIN AND METALLOPROTEASE (ADAM) 33 PROTEIN IN PATIENTS WITH PULMONARY SARCOIDOSIS}

doi:10.1136/thx.2010.150946.42

${ }^{1} \mathrm{~A} S$ Shaffiq, ${ }^{1} \mathrm{H} \mathrm{M} H$ Haitchi, ${ }^{1} \mathrm{Y} Y \mathrm{P}$ Pang, ${ }^{2} \mathrm{~A}$ A Alangari, ${ }^{1} \mathrm{M} \mathrm{G} J$ Jones, ${ }^{1} \mathrm{~B}$ G M Marshall, ${ }^{1} \mathrm{~K}$ M A 0 O'Reilly, ${ }^{1} \mathrm{D}$ E D Davies. ${ }^{1}$ University of Southampton School of Medicine, Southampton, UK; ${ }^{2}$ King Saud University, Riyadh, Saudi Arabia

Background The asthma and chronic obstructive pulmonary disease (COPD) gene, A Disintegrin And Metalloproteinase (ADAM)33, is selectively expressed in mesenchymal cells and its metalloprotease activity has been linked to angiogenesis and airway remodelling. A soluble form of ADAM33 (sADAM33) has been identified in the bronchoalveolar lavage fluid (BALF) of asthmatic patients and its levels inversely correlate with lung function and disease severity. Because tissue remodelling also occurs in pulmonary sarcoid, we hypothesised that sADAM33 is elevated in BALF of patients with this disease which, like asthma, is heterogeneous.

Methods BALF was obtained from healthy controls $(n=11)$ and patients with sarcoid $(n=13)$ using fibre optic bronchoscopy according to current guidelines. After removal of immunoglobulins using Protein $\mathrm{A} / \mathrm{G}$ and enrichment using Concanavalin $\mathrm{A}$ beads, sADAM33 was identified in BALF by Western blotting. A FRET peptide cleavage assay was used to assess ADAM33-like activity in BALF. Lung function (FVC\%) and gas transfer (TLCO\%) were measured at time of first diagnostic workup.
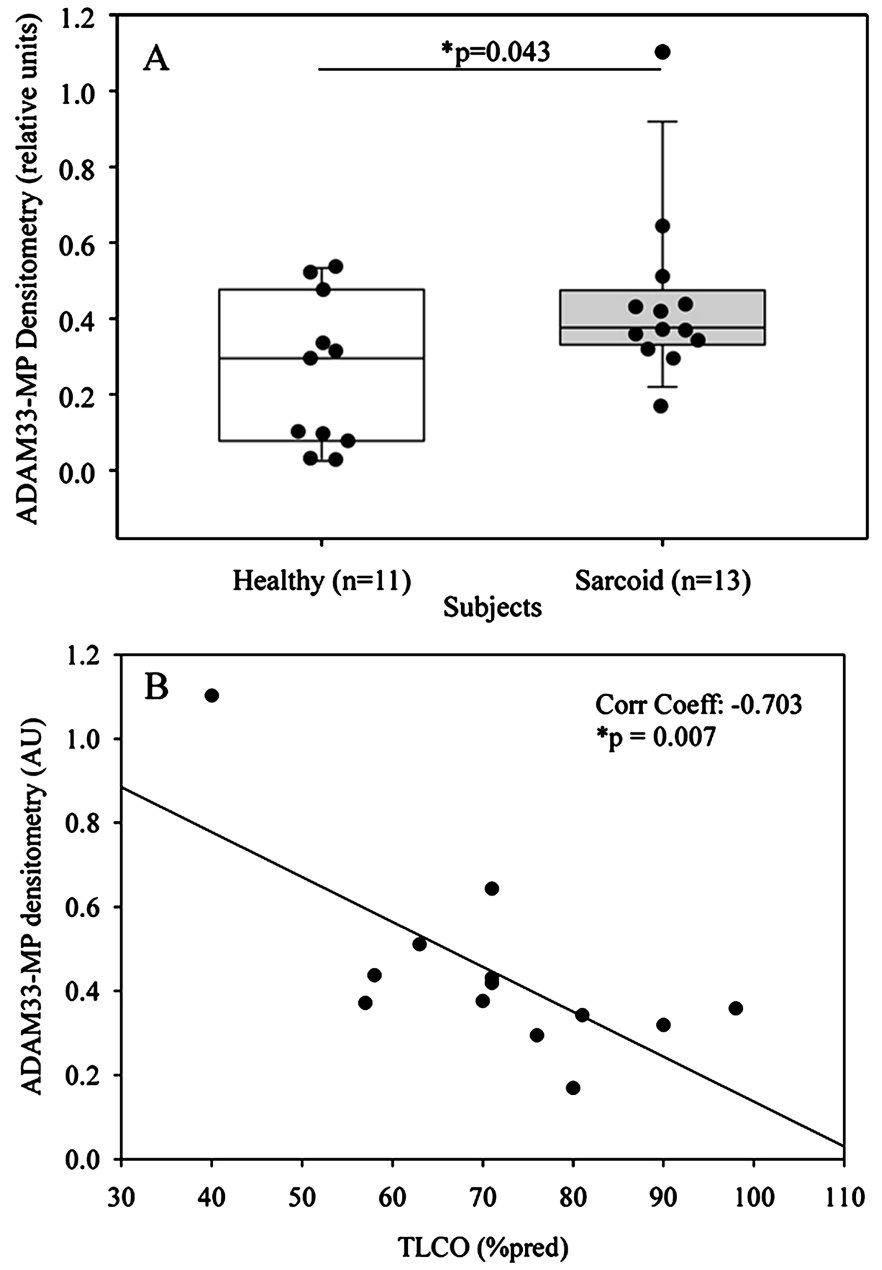

Abstract S141 Figure 1

Results sADAM33 protein in BALF was detected as a $25 \mathrm{kDa}$ fragment and levels were significantly increased in samples from sarcoid patients when compared to healthy controls $(p<0.05)$ (Abstract S141 Figure 1A). Levels of sADAM33 were inversely correlated with lung function (FVC $\%$ predicted) $(\mathrm{p}<0.05)$ and gas transfer $($ TLCO $\%$ predicted) $(p<0.01)$ (Abstract S141 Figure 1B). No difference in sADAM33 enzymatic activity was observed between healthy and sarcoid BALF samples.

Conclusion Release of sADAM33 is increased in sarcoid in association with abnormal lung function. Further studies will be required to determine whether the release of sADAM33 results in dysregulated metalloprotease activity, leading to angiogenesis and pulmonary parenchymal remodelling in pulmonary sarcoid. Since ADAM33 polymorphism is related to reduced lung function in asthma and COPD, this study raises the possibility that there may also be genetic associations between $A D A M 33$ and some forms of pulmonary sarcoid. Finally, the occurrence of sADAM 33 in asthma and sarcoidosis and its relation to reduced lung function suggests that it may be a biomarker of pulmonary remodelling in these diseases.

\section{S142 THE $\mathrm{K}^{+}$CHANNEL KCa3.1 IS EXPRESSED IN HUMAN LUNG FIBROBLASTS}

doi:10.1136/thx.2010.150946.43

${ }^{1} \mathrm{~K}$ Roach, ${ }^{1} \mathrm{G}$ Arthur, ${ }^{2} \mathrm{C}$ Feghali-Bostwick, ${ }^{3} \mathrm{~W}$ Coward, ${ }^{1} \mathrm{~S} \mathrm{M}$ Duffy, ${ }^{1} \mathrm{P}$ Bradding ${ }^{1}$ Institute for Lung Health, Department of Infection, Immunity and Inflammation, University of Leicester, Leicester, UK; ${ }^{2}$ Department of Medicine, Division of Pulmonary, Allergy, and Critical Care Medicine, University of Pittsburgh, Pittsburgh, USA; ${ }^{3}$ Division 